\title{
Rats seem indifferent between their own scent-marked homecages and clean cages
}

\author{
Charlotte C. Burn ${ }^{\mathrm{a}, *}$, Georgia J. Mason ${ }^{\mathrm{b}}$ \\ ${ }^{a}$ Department of Zoology, University of Oxford, South Parks Road, Oxford OX1 3PS, UK \\ ${ }^{\mathrm{b}}$ Department of Animal \& Poultry Science, University of Guelph, Ontario N1G 2W1, Canada \\ Accepted 4 June 2008 \\ Available online 17 July 2008
}

\begin{abstract}
Previous large-scale, long-term studies suggest that rat welfare is little affected by cage-cleaning frequency. Here, we investigate this further using a preference test: arguably a more sensitive welfare indicator than those used previously. Nine pairs of rats were each housed in two interconnected cages of differing cleanliness. One cage was cleaned every 3-4 days, while the other remained uncleaned for 18 days. All rats were handled at each cage-cleaning (regardless of which cage they chose) so that this aspect of the cleaning routine remained consistent. Furthermore, ammonia build-up was negligible. We could thus see whether rats prefer their own scent-marks over clean bedding, without the confounds of differential handling or the potential harm induced by ammonia. Dwelling, resting, feeding, drinking, defecation, and ammonia concentrations were compared between the two cages. None were found to differ significantly between the cages over the course of the experiment. Power tests and confidence intervals support the conclusion that the rats had no meaningful preference for self-scented, familiar areas over clean areas. A weak preference cannot be ruled out, due to the small sample and the space limitations within laboratory cages. Also, the lack of ammonia means that the threshold at which rats would start to avoid soiled areas that do generate this compound remains undetermined. Nevertheless, together with previous studies showing no clear effects of cage-cleaning frequency on rat welfare, these choice tests indicate that it is unlikely that olfactory disruption during cage-cleaning is an important welfare concern for stable nonbreeding groups of rats.
\end{abstract}

(C) 2008 Elsevier B.V. All rights reserved.

Keywords: Animal welfare; Husbandry; Hygiene; Olfaction; Preference tests; Rats

* Corresponding author at: School of Clinical Veterinary Science, University of Bristol, Langford House, Bristol BS40 5DU, UK.

E-mail address: charlotte.burn@worcester.oxon.org (C.C. Burn).

0168-1591/\$ - see front matter (C) 2008 Elsevier B.V. All rights reserved.

doi:10.1016/j.applanim.2008.06.002 


\section{Introduction}

Previous research investigating how cage-cleaning frequency affects laboratory rat welfare suggests that rats fare similarly whether under twice-weekly cleaning regimes, or when their cages are cleaned only every 2 weeks. In a 5-month long study, cleaning frequencies were compared in terms of rats' acute behavioural and chromodacryorrhoea (an aversion-related Harderian gland secretion) responses to cleaning (Burn et al., 2006b), and their long-term aggressiveness, health, chromodacryorrhoea, handleability, and lung and adrenal pathology (Burn et al., 2006a). In each case, cleaning frequency showed no clear effects on rat welfare. Furthermore, no significant effects were found in a longitudinal study investigating how pre-weaning cage-cleaning frequency affected rats' later anxiety profiles as adults (Burn et al., 2008). The one exception was that, in breeding rats, more frequent cage-cleaning slightly but significantly increased the risk that pups would be cannibalised (Cisar and Jayson, 1967; Burn and Mason, 2008); this effect appeared to be due to disturbances being more likely to occur when pups were newborn and vulnerable if cleaning was more frequent, not due to a cumulative effect on dam welfare.

Any proposed distress from cage-cleaning could be caused by disruption of the olfactory environment (Jennings et al., 1998; Koolhaas, 1999; Hansen et al., 2000), or the disturbances associated with being transferred between cages, including handling (Balcombe et al., 2004), exposure to brighter light, and increased sound levels (Gamble, 1982; Voipio et al., 2006). Here, we concentrate on the first of these possibilities: olfactory disruption. Juvenile rats appear less anxious on self-soiled bedding than on clean bedding (Adams et al., 1983; Richardson and Campbell, 1988), but whether or not the same applies for adult rats is unknown. There must presumably be a threshold above which the degree of soiling becomes excessive and potentially harmful, when rats should start to prefer clean bedding. The (space) beststudied component of cage-soiling is ammonia, although the rat's tolerance of ammonia relative to humans is not yet known. Concentrations above $100 \mathrm{ppm}$ have occasionally been observed in artificial rat burrows (Studier and Baca, 1968), but can increase blinking (Broderson et al., 1976), decrease activity levels (Tepper et al., 1985), and cause respiratory problems (Serrano, 1971; Broderson et al., 1976; Gamble and Clough, 1976; Schoeb et al., 1982; Bolon et al., 1991). Lower concentrations, which are more representative of current in-cage concentrations (Hoglund and Renstrom, 2001; Burn et al., 2006a; Burn and Mason, 2008), have not been tested in rats, but mice show no significant preference or avoidance of them (Green et al., 2008). Here, we selected an animal unit known to produce low concentrations of ammonia, allowing us to investigate rats' preferences for clean or scentmarked cages over time, without the confound of ammonia building up to harmful or aversive concentrations.

In this experiment, we also separated rats' preferences for clean or soiled cages from the other disturbances associated with cage-cleaning. Behavioural observations were taken during the light and dark phases, to monitor the rats' general preferences over the whole circadian period, and a baseline was recorded when the cages did not differ (Blom et al., 1993, 1995). We used socially housed rats, not only because rats should be housed socially whenever possible (e.g. Hurst et al., 1998; Patterson-Kane et al., 2002; Sharp et al., 2002), but also because the potential welfare impacts of different in-cage olfactory environments might include social effects (e.g. territorial security or aggression). 


\section{Methods}

\subsection{Animals and housing}

Adult hooded Lister rats were housed in 10 single-sex pairs ( 7 male and 3 female pairs), in an animal room known to produce low concentrations of ammonia. Each pair was housed in two $0.6 \mathrm{~m}$ $\times 0.3 \mathrm{~m} \times 0.3 \mathrm{~m}$ cages joined together (Fig. 1). Both cages included a food-hopper, a drinking bottle, a flannel hammock, strips of paper nesting material, a woollen sock, and a cardboard tube. The rats were provided with food (RM3 pelleted diet, Special Diet Services) and water ad libitum, and their diet was supplemented twice weekly by scattering a seed and dried fruit mix for foraging. The bedding was aspen woodchips, grade 8 (Lillico, Surrey, UK) to a depth of $2 \mathrm{~cm}$. The temperature and humidity were $22{ }^{\circ} \mathrm{C}$ and $50 \%$, respectively. The light:dark schedule was 12:12, with lights on at 3 a.m.; this time-shift was introduced gradually from 7 a.m. over 10 days and had remained stable for 2 days before the experiment. The unfamiliar observer stood in the room making notes to habituate the rats to the observation procedure for 30 90 min on four occasions during the week preceding the experiment.

\subsection{Cleaning routine}

On day 0 , both cages were cleaned, but thereafter only the 'clean' cage was cleaned twice weekly. The 'non-cleaned' cage was left undisturbed for 18 days. Half the non-cleaned cages were positioned on the left of the clean cages, and half on the right. During cleaning, both rats were transferred into a holding cage for approximately $3-5 \mathrm{~min}$. The bedding was removed from the clean cage, the cage was washed with Virkon ${ }^{\mathrm{TM}}$ solution, dried with paper towels, and fresh bedding was added. The enrichment items and cage lid remained unchanged. A handful of fresh forage was added to both cages after cleaning.

\subsection{Measurements and observations}

At the start of the experiment $700 \mathrm{~g}$ of food was provided in each hopper, and the water-level in each bottle was marked using an indelible pen. Then, immediately before each cage-cleaning, photographs were taken of both cages for later scoring, the food in each hopper was weighed, and the new water-level was recorded and re-marked. Ammonia concentrations were also measured using a pump with glass tubes that detected ammonia at either 2-30 or 5-100 ppm (Shawcity Ltd., Oxfordshire, UK). Tubes were held about $5 \mathrm{~cm}$ above the bedding, while cages remained in situ.

Behaviour was observed 1 day after each cleaning event (to avoid novelty effects and disrupted behaviour associated with cleaning; Saibaba et al., 1996; Schnecko et al., 1998; Duke et al., 2001; Sharp et al., 2002; Burn et al., 2006b). Instantaneous observations were taken every $10 \mathrm{~min}$ for two 2-h periods during the light phase (from 10:00 and from 13:00), and then for one 2-h period during the dark phase (from 16:00). Rats were allowed to habituate to the observer's presence for $10 \mathrm{~min}$ before observations started. Very dim white light (provided by a commercially available night-light), which the rats were habituated to, was used to observe them during the dark period.

Three behaviours were observed sufficiently often for statistical analysis: dwelling frequency (i.e. presence of one or both rats) in each cage; which cage rats chose to rest in when they rested directly on the bedding; and, when in the non-cleaned cage, the proportion of resting that was in the hammock. Resting was defined as lying down, moving very little, with eyes closed or half-closed. Also, food and water consumption were analysed, and faecal pellets were counted from the photographs. Social behaviours including aggression and allogrooming were of interest, but were observed too rarely for analysis.

\subsection{Statistical analyses}

The two observation periods during the light (inactive) phase were pooled to compensate for the longer bout durations associated with resting, which might otherwise have indicated a stronger preference for one 
(A)
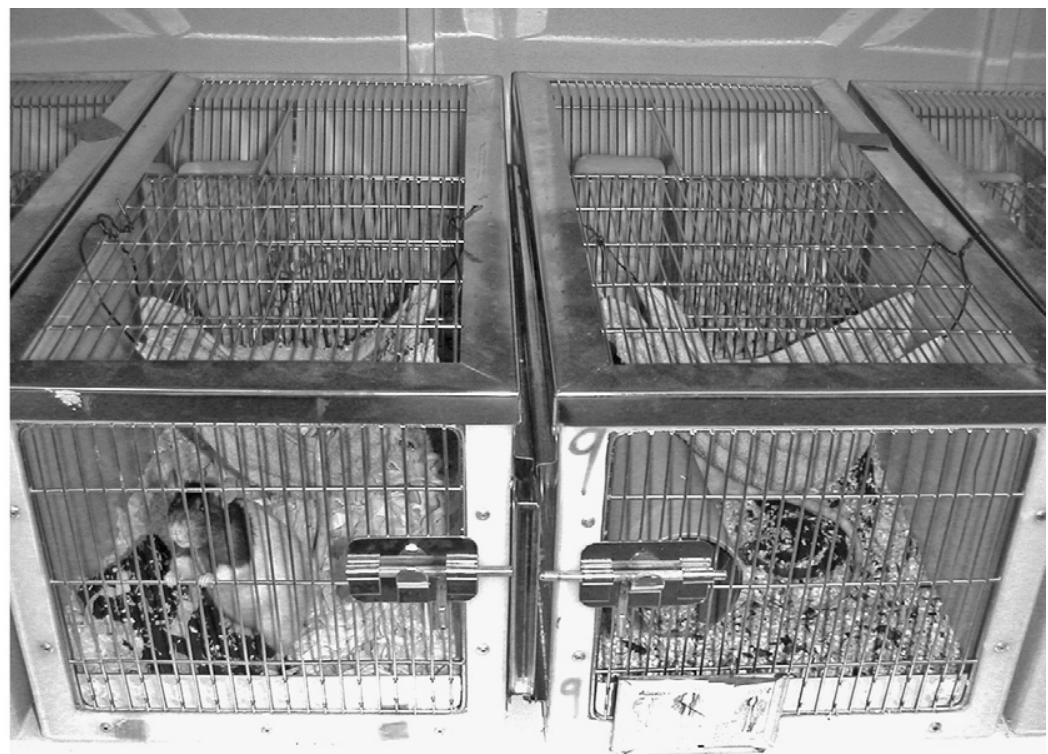

(B)

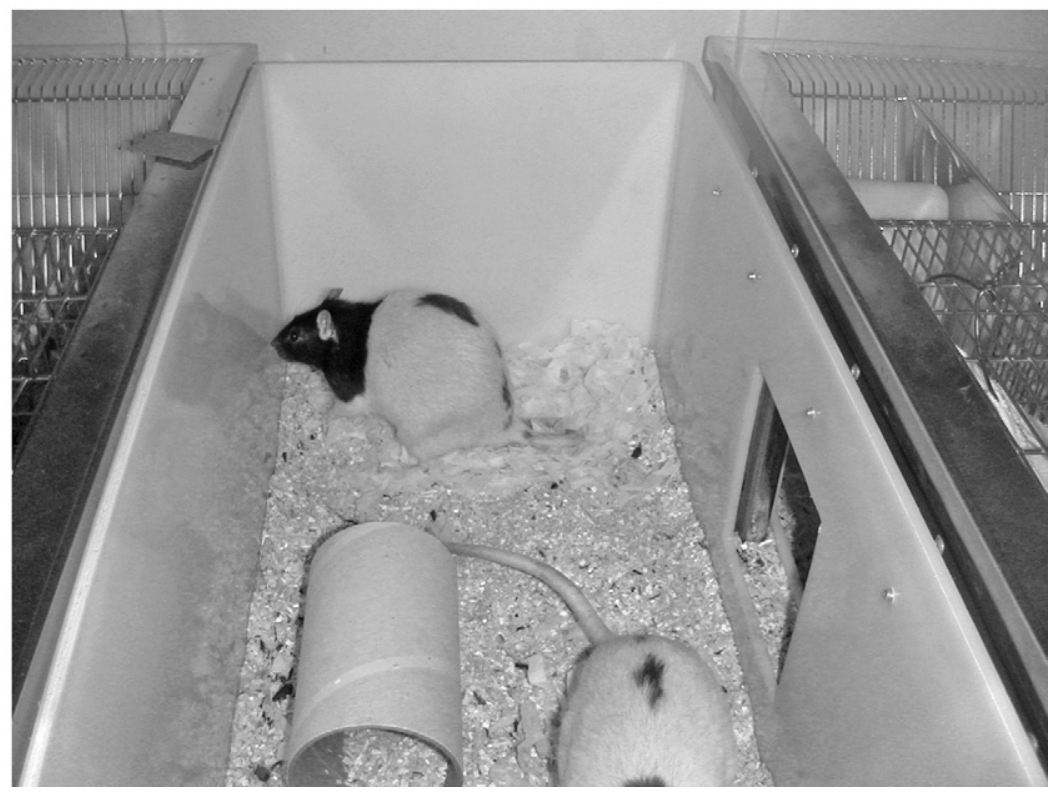

Fig. 1. The paired cages (A). The two rats were able to move freely between both sides via an opening in the central partition, shown in (B) with the lid removed. One cage was cleaned every 3-4 days, and the other remained undisturbed for 18 days, but the cages were otherwise treated identically.

cage than if the rats had moved between the cages more actively. The final sample size was nine pairs because one rat became ill, so that pair was excluded from analyses.

The proportion of each activity taking place in the non-cleaned cage was used as the $y$-variable, except for hammock-use in the non-cleaned cage, where the proportion of resting in the hammock versus the bedding was used. Repeated measures general linear models (GLMs) were used, with the pair of rats (a 
random factor), day, and for behavioural observations, circadian period (light or dark) as predictors. Day was included as a covariate unless graphical inspection of the data suggested a non-linear relationship (e.g. if soiling above a certain threshold caused a behavioural change), when it was reanalysed as categorical. In some analyses, specific behaviours were also included in case they influenced each other; for example, numbers of new faecal pellets could have influenced time spent resting on the bedding. Residuals were assessed graphically to assess model fit, and data were arc-sine transformed where necessary. The small sample size meant that sex could not be included, so any sex differences would have contributed to the variation between pairs.

Confidence intervals are suggested to be more informative than post hoc power tests for estimating detectable effect sizes (Colegrave and Ruxton, 2003), so these were calculated from the final day of the experiment, when we would expect to see the strongest preference in either direction. One-sample $t$-tests were used to calculate $95 \%$ confidence intervals, comparing the proportions of behaviour in the non-cleaned cage against the null hypothesis of no preference. Post hoc power tests have been criticised because conclusions about the null hypothesis from the $P$-value and from power tests are contradictory (Hoenig and Heisey, 2001), but because our sample size was small, they are used here as an additional tool to suggest approximate detectable effect sizes. No entirely appropriate power tests existed for the experimental design (Bausell and $\mathrm{Li}, 2002$ ), so power tests for one-sample $t$-tests were used. The power was set to 0.8 , the sample size was 9 , and alpha was 0.05 . Our repeated measures design will have reduced the variation contributed by the pairs of rats themselves, so standard deviations were calculated from the residuals of GLMs that tested the effects of the pair of rats and the circadian period. This gave a measure of the variation remaining after taking into account the individual pairs and the circadian period. Only standard errors from the first day of measurements, when the two cages differed least, were used.

\section{Results}

No ammonia was detected at any time. The equipment was subsequently tested in other laboratories and using pure ammonium chloride, and it functioned effectively in those situations. Furthermore, the cages did not subjectively smell of ammonia, although they did have a strong general smell by Days 15 and 18. Fig. 2 shows the accumulation of faecal pellets over time, and illustrates the divergence between clean and dirty cages.

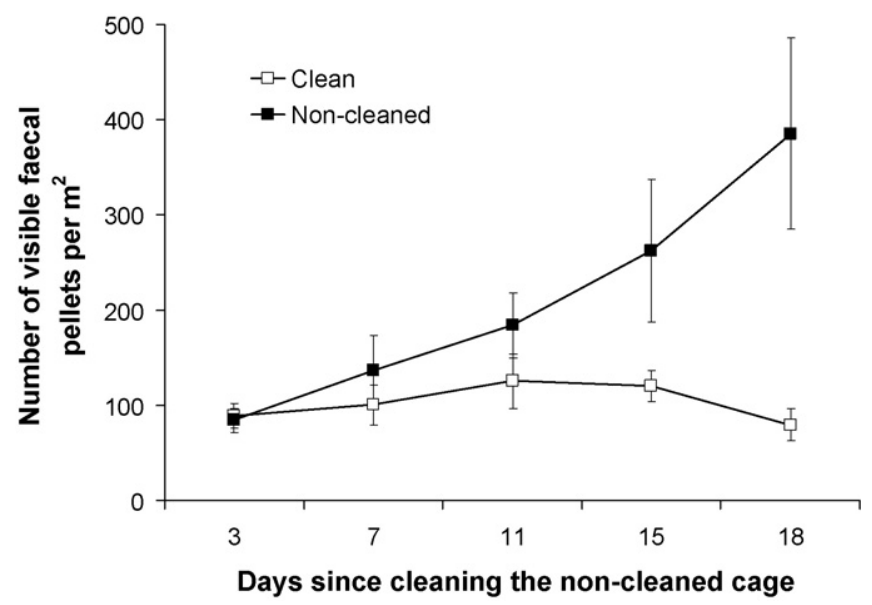

Fig. 2. Density of visible faecal pellets (mean \pm S.E.) in the two cages over the period of the experiment. Faecal pellets were counted from photographs taken immediately before cage-cleaning. 
Over time, there were no significant differences, nor consistent trends between the treatments for any of the measurements of rat behaviour, even on the final day when the difference between the two cages was at its greatest (Fig. 3). The observed non-significant $P$-values and the estimated detectable effect sizes are shown in Table 1.

Individual pairs of rats consistently dwelled $\left(F_{8,96}=6.74 ; P=<0.001\right)$ and rested $\left(F_{8,111}=8.83 ; P=<0.001\right)$ in one particular cage, despite there being no population-wide
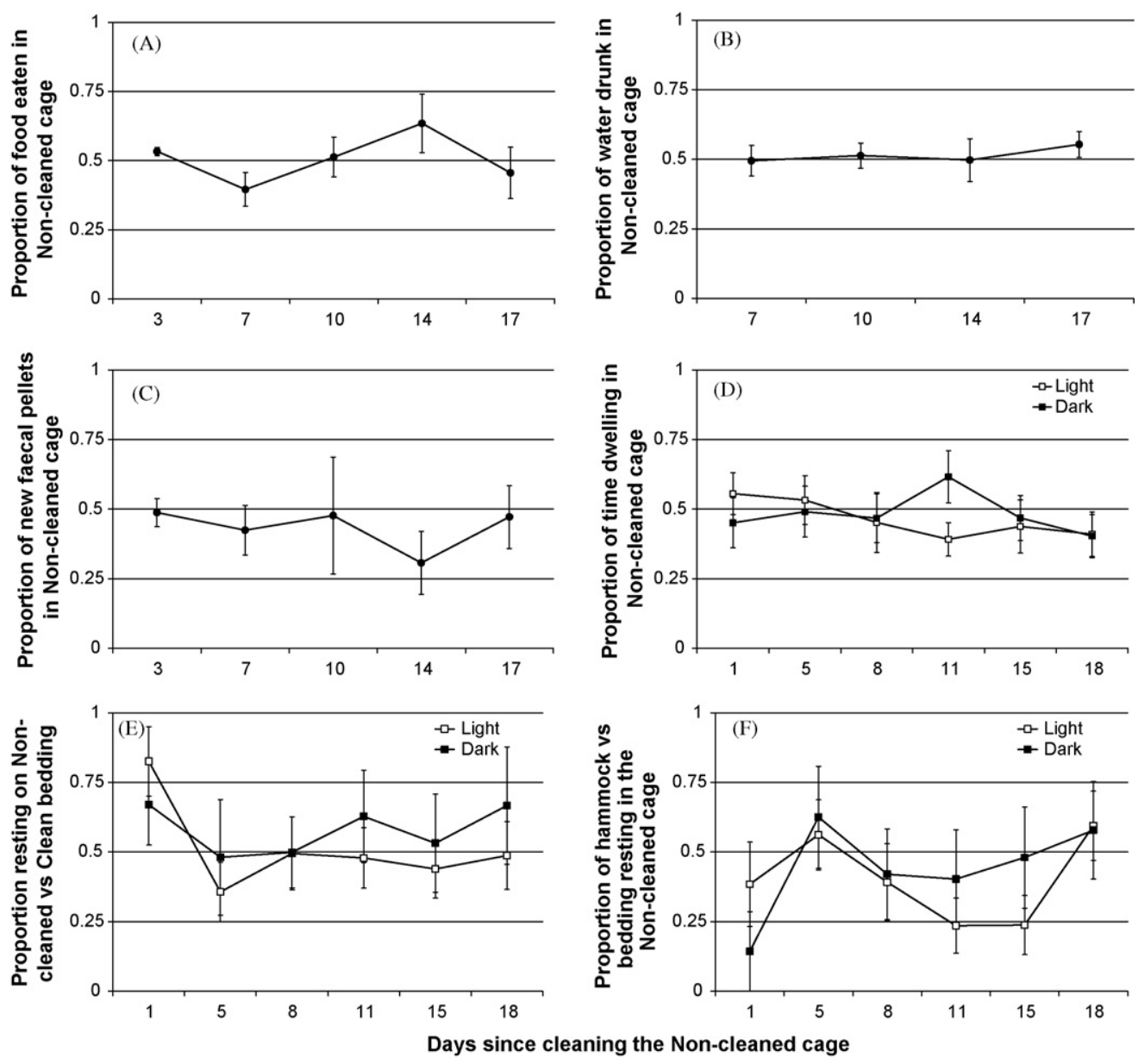

Fig. 3. Proportions (mean \pm S.E.) of the following activities that took place in the non-cleaned cage during the 18 days that it was left undisturbed. The proportion of (A) food eaten, (B) water consumed, and (C) new faecal pellets in the noncleaned cage are shown, as well as the proportion of time spent (D) dwelling and (E) resting on the bedding in the noncleaned cage. Graph $(\mathrm{F})$ shows the proportion of time spent resting in the hammock rather than on the bedding when in the non-cleaned cage. Graphs (D-F) show observations that were replicated during the light and dark phases. The first measurement was taken when there was no difference between the cleanliness of the two cages (except (B) drinking, because the first measurement failed). A proportion of 0.5 indicates no preference, shown by the horizontal line, except in Graph F, where a lack of preference would lie around 0.4 (the baseline calculated on Day 1). If the rats had preferred noncleaned cages, we would expect an upwards trend in all graphs, except Graph F, where there might have been a negative trend; the reverse should have been observed if they had preferred clean cages. In fact there were no significant changes over time for any of the measurements. 
Table 1

The statistical significance, standard deviations, and estimated detectable effect sizes for rats' preferences for a clean or a non-cleaned cage

\begin{tabular}{llllll}
\hline $\begin{array}{l}\text { Proportion in } \\
\text { non-cleaned cage }\end{array}$ & $\begin{array}{l}\text { Observed } \\
P \text {-value }\end{array}$ & $\begin{array}{l}\text { Standard } \\
\text { deviation }\end{array}$ & $\begin{array}{l}\text { Expected } \\
\text { proportion } \\
\text { under } \mathrm{H}_{0}\end{array}$ & $\begin{array}{l}\text { Detectable effect } \\
\text { size with a } \\
\text { power of 0.8 }\end{array}$ & $\begin{array}{l}\text { Confidence } \\
\text { interval } \\
\text { for Day 18 }\end{array}$ \\
\hline Feeding & 0.640 & 0.17 & 0.50 & $0.33-0.67$ & $0.24-0.67$ \\
Drinking & 0.436 & 0.14 & 0.50 & $0.35-0.65$ & $0.44-0.66$ \\
Dwelling & 0.153 & 0.22 & 0.50 & $0.27-0.73$ & $0.24-0.57$ \\
Faecal pellets & 0.509 & 0.21 & 0.50 & $0.27-0.73$ & $0.21-0.73$ \\
Resting in hammock* & 0.676 & 0.25 & $0.40^{\dagger}$ & $0.13-0.77$ & $0.22-0.92$ \\
Resting on bedding & 0.249 & 0.29 & 0.50 & $0.18-0.82$ & $0.21-0.99$ \\
\hline
\end{tabular}

The level of statistical significance was set to 0.05 . The standard deviations were calculated from the first day of data collection, using the residuals of a GLM that accounted for variation from the pairs of rats, and the circadian period. The effect size was calculated for a sample size of nine and a power of 0.8 . For all variables the effect size represents the proportion of the observations that would have to occur in either cage for a significant preference to be detected with the desired power; the exception is resting in the hammock*, where it represents the proportion of resting on either the noncleaned bedding or the hammock that would have shown a detectable preference. The confidence intervals are calculated using a one-sample $t$-test comparing data from Day 18 against the proportion of observations expected to occur in the noncleaned cage under the null hypothesis $\left(\mathrm{H}_{0}\right) . \mathrm{H}_{0}$ is 0.5 , except ${ }^{\dagger}$ when it was the proportion observed on Day 1.

preference. Rats spent similar proportions of time together in the same cage as they spent apart in different cages (mean \pm S.E. proportion of time together on Day $1=0.56 \pm 0.06 ; n=9$; $P=0.367)$.

\section{Discussion}

Here we aimed to assess whether rats preferred cleaner cages or soiled cages that smelled familiar, while the cage-cleaning frequency itself (including aspects such as handling and increased noise levels) was kept constant; and while ammonia build up was minimised. We chose to use a laboratory with low ammonia production. The complete lack of ammonia was somewhat unexpected, although in another laboratory within the same building, cages housing four rats usually generated no detectable ammonia after 13 days (Burn et al., 2006a). Its absence might mean that bacterial growth within the cages here was relatively slow compared with other laboratories (e.g. Hawkins et al., 2003; Burn et al., 2006a), and the degree of soiling might have been intrinsically less harmful to rat health (Schoeb et al., 1982).

If rats had preferred their own familiar scent, then these innocuous non-cleaned cages should perhaps have provided the ideal environment (assuming that, like mice (Green et al., 2008), rats are not positively attracted towards ammonia). No such preference for the scent-marked cage was observed, nor even a trend towards it. Indeed the rats showed no significant preferences or behavioural differences between the two cages-even on the last day when the cages differed most. Thus the example graphs in Fig. 3 show no consistent changes in the rats' use of the noncleaned cage over the 18 days of the experiment. If they had preferred soiled cages, we would expect a positive trend in all graphs, except for Fig. 3F which would have shown a negative trend as rats increasingly rested on the soiled bedding rather than the hammock. The sample size was small, but it was sufficient to detect moderate preferences (proportions of 0.63-0.73) for four of our six measures (Table 1). For dwelling preferences for example, the confidence interval shows that - even on Day 18 - we can be $95 \%$ certain that pairs of rats spent less than a mean of $57 \%$ of their time in the non-cleaned cage (Table 1). It seems therefore that the derogatory phrase 'dirty 
rat' (oft-misquoted from James Cagney in the 1932 film, 'Taxi'; Cagney, 1976) is inaccurate, since rats do not prefer to inhabit soiled areas.

One consideration is whether the olfactory differences between the two cages could have been obscured by the close proximity of the cages to each other, and by the retention of the cage lid and enrichment items over the study period. The cages might then have been more difficult to discriminate than if the clean cage was completely void of all scent marks - volatile and involatile - after each cleaning event. However, the retention of the lid and/or enrichments mimics the cleaning regime in many laboratories (e.g. Burn et al., 2006a; Schondelmeyer et al., 2006; AbouIsmail et al., 2008; Burn and Mason, 2008), so this study models what would happen in these laboratories at least. Moreover, to the human observer (CB), the two cages subjectively smelled noticeably different to each other during the final week of the study; and given that olfaction in rats is vastly more sensitive than in humans (Burn, 2008), this difference would probably have been more obvious to them and detectable earlier in the study.

Overall, these results therefore suggest that removing non-breeding rats' soiled bedding during cage-cleaning causes them no serious welfare problems, which is in agreement with our previous large-scale studies (Burn et al., 2006a,b) (but for breeding rats, see Burn and Mason, 2008). It should be noted that the rats could still have had a subtle preference for either cage, because a larger sample size would be necessary to detect more subtle preferences than those detectable here. However, whether a subtle preference is meaningful in terms of being a welfare priority is questionable, given the more serious welfare requirements that still require increased recognition, such as social housing (e.g. Hurst et al., 1998; Patterson-Kane et al., 2002; Sharp et al., 2002), environmental enrichment (e.g. Patterson-Kane, 2001; Olsson et al., 2003; Burman et al., 2006), and reduction of stress and pain in procedures (Balcombe et al., 2004; Richardson and Flecknell, 2005).

If future work was conducted, we would suggest the following. First, for the two resting behaviours only strong effects would have been detectable with our sample size. The high variance probably resulted from the rats using one cage as their homecage (in agreement with Blom et al., 1995), so for a significant change, rats' preferences would have to be strong enough to cause them to switch away from their chosen homecage. It could therefore be worthwhile to repeat this aspect of the study with a larger sample size. Second, it is possible that the paired rats influenced each other's preferences, although they showed no significant avoidance of or attraction to each other. It would be interesting to know the dominance relationships of the pairs, in case the dominants and subordinates had different preferences for the olfactory status of the cages, as seen in mice (Fitchett et al., 2006). Third, we learned little about when rats would start to avoid their soiled cages, given the opportunity, if ammonia did start to accumulate, and this still remains an unknown issue. While concentrations above $100 \mathrm{ppm}$ can cause health problems as described above (Serrano, 1971; Broderson et al., 1976; Gamble and Clough, 1976; Schoeb et al., 1982; Tepper et al., 1985; Bolon et al., 1991), modern in-cage concentrations tend to be much lower (Hoglund and Renstrom, 2001; Burn et al., 2006a; Burn and Mason, 2008). In-cage ammonia concentrations ranging between 0 and $85 \mathrm{ppm}$ showed no relationship with respiratory pathology or sneezing rates (Burn et al., 2006a), but no more subtle evaluation of rats responses to low ammonia concentrations has yet been published, as it has for mice (Green et al., 2008).

\section{Conclusions}

Despite the potential conflict between rats communicating using scent and yet relying on us to keep their cages hygienic, when given the choice rats showed no significant preference for 
homecages marked with their own scent. A weak preference cannot be ruled out, but the results suggest that the olfactory disruption caused by cage-cleaning has little impact on experimental rat welfare.

\section{Acknowledgements}

Many thanks to R. Clubb and V. Clark for carrying out the husbandry for this experiment. This work was funded by the Animal Procedures Committee of the Home Office, UK.

\section{References}

Abou-Ismail, U.A., Burman, O.H.P., Nicol, C.J., Mendl, M., 2008. Let sleeping rats lie: does the timing of husbandry procedures affect laboratory rat behaviour, physiology and welfare? Appl. Anim. Behav. Sci. 111, 329-341.

Adams, J., Miller, D.R., Nelson, C.J., 1983. Ultrasonic vocalizations as diagnostic tools in studies of developmental toxicity: an investigation of the effects of prenatal treatment with methylmercuric chloride. Neurobehav. Toxicol. Teratol. 5, 29-34.

Balcombe, J.P., Barnard, N.D., Sandusky, C., 2004. Laboratory routines cause animal stress. Contemp. Top. Lab. Anim. Sci. 43, 42-51.

Bausell, R.B., Li, Y.-F., 2002. Power Analysis for Experimental Research: A Practical Guide for the Biological, Medical and Social Sciences. Cambridge University Press, Cambridge.

Blom, H.J.M., Baumans, V., Van Vorstenbosch, C.J.A.H.V., Van Zutphen, L.F.M., Beynen, A.C., 1993. Preference tests with rodents to assess housing conditions. Anim. Welf. 2, 81-87.

Blom, H.J.M., Van Tintelen, G., Baumans, V., Van Den Broek, J., Beynen, A.C., 1995. Development and application of a preference test system to evaluate housing conditions for laboratory rats. Appl. Anim. Behav. Sci. 43, 279-290.

Bolon, B., Bonnefoi, M.S., Roberts, K.C., Marshall, M.W., Morgan, K.T., 1991. Toxic interactions in the rat nose: pollutants from soiled bedding and methyl bromide. Toxicol. Pathol. 19, 571-579.

Broderson, J., Lindsey, J., Crawford, J., 1976. The role of environmental ammonia in respiratory mycoplasmosis of rats. Am. J. Pathol. 85, 115-130.

Burman, O.H.P., Abou-Ismail, U.A., Nicol, C.J., Day, M., Owen, D., Bailey, M., Mendl, M., 2006. A multidisciplinary study of the long-term effects of environmental enrichment on laboratory rat welfare. In: Paper presented at the 40th International Congress of the ISAE, Bristol.

Burn, C.C., 2008. What is it like to be a rat? Rat sensory perception and its implications for experimental design and rat welfare. Appl. Anim. Behav. Sci. 112, 1-32.

Burn, C.C., Mason, G.J., 2008. Effects of cage-cleaning frequencies on rat reproductive performance, infanticide, and welfare. Appl. Anim. Behav. Sci. 114, 235-247.

Burn, C.C., Day, M.J., Peters, A., Mason, G.J., 2006a. Long-term effects of cage-cleaning frequency and bedding type on laboratory rat health, welfare, and handleability: a cross-laboratory study. Lab. Anim. 40, 353-370.

Burn, C.C., Peters, A., Mason, G.J., 2006b. Acute effects of cage cleaning at different frequencies on laboratory rat behaviour and welfare. Anim. Welf. 15, 161-172.

Burn, C.C., Deacon, R., Mason, G.J., 2008. Marked for life? Effects of early cage cleaning frequency, delivery batch and identification tail-marking on adult rat anxiety profiles. Dev. Psychobiol. 5, 266-277.

Cagney, J., 1976. Cagney by Cagney. Doubleday, New York.

Cisar, C.F., Jayson, G., 1967. Effects of frequency of cage cleaning on rat litters prior to weaning. Lab. Anim. Care 17, 215-217.

Colegrave, N., Ruxton, G.D., 2003. Confidence intervals are a more useful complement to nonsignificant tests than are power calculations. Behav. Ecol. 14, 446-447.

Duke, J., Zammit, T., Lawson, D., 2001. The effects of routine cage-cleaning on cardiovascular and behavioural parameters in male Sprague-Dawley rats. Contemp. Top. Lab. Anim. Sci. 40, 17-20.

Fitchett, A.E., Barnard, C.J., Cassaday, H.J., 2006. There's no place like home: cage odours and place preference in subordinate CD-1 male mice. Physiol. Behav. 87, 955-962.

Gamble, M.R., 1982. Noise and laboratory animals. J. Inst. Anim. Tech. 33, 5-15.

Gamble, M.R., Clough, G., 1976. Ammonia build-up in animal boxes and its effect on rat tracheal epithelium. Lab. Anim. 10, 93-104. 
Green, A.R., Wathes, C.M., Demmers, T.G.M., Clark, J.M., Xin, H.W., 2008. Development and application of a novel environmental preference chamber for assessing responses of laboratory mice to atmospheric ammonia. J. Am. Assoc. Lab. Anim. Sci. 47, 49-56.

Hansen, A.K., Baumans, V., Elliott, H., Francis, R., Holgate, B., Hubrecht, R., Jennings, M., Peters, A., Stauffacher, M., 2000. Future principles for housing and care of laboratory rodents and rabbits (second meeting). Strasbourg: Report of the Working Party for the Preparation of the Fourth Multilateral Consultation of Parties to the European Convention for the Protection of Vertebrate Animals used for Experimental and Other Scientific Purposes.

Hoenig, J.M., Heisey, D.M., 2001. The abuse of power: the pervasive fallacy of power calculations for data analysis. Am. Statistician 55, 19-24.

Hoglund, A.U., Renstrom, A., 2001. Evaluation of individually ventilated cage systems for laboratory rodents: cage environment and animal health aspects. Lab. Anim. 35, 51-57.

Hurst, J.L., Barnard, C.J., Nevison, C.M., West, C.D., 1998. Housing and welfare in laboratory rats: the welfare implications of social isolation and social contact among females. Anim. Welf. 7, 121-136.

Jennings, M., Batchelor, G.R., Brain, P.F., Dick, A., Elliott, H., Francis, R.J., Hubrecht, R.C., Hurst, J.L., Morton, D.B., Peters, A.G., Raymond, R., Sales, G.D., Sherwin, C.M., West, C., 1998. Report of the rodent refinement working party. Refining rodent husbandry: the mouse. Lab. Anim. 32, 233-259.

Koolhaas, J.M., 1999. The laboratory rat. In: Poole, T. (Ed.), The UFAW Handbook on the Care and Management of Laboratory Animals: Terrestrial Vertebrates, 7th ed., vol. 1. Blackwell Science, Oxford, pp. 313-330.

Hawkins, P., Anderson, D., Applebee, K., Key, D., Wallace, J., Milite, G., MacArthur Clark, J., Hubrecht, R., Jennings, M., 2003. Individually ventilated cages and rodent welfare: Report of the 2002 RSPCA/UFAW rodent welfare group meeting. Anim. Technol. Welf. 2, 23-34.

Olsson, I.A.S., Nevison, C.M., Patterson-Kane, E.G., Sherwin, C.M., Van de Weerd, H.A., Wurbel, H., 2003. Understanding behaviour: the relevance of ethological approaches in laboratory animal science. Appl. Anim. Behav. Sci. 81, 245-264.

Patterson-Kane, E.G., 2001. Environmental enrichment for laboratory rats: a review. Anim. Technol. 52, 77-84.

Patterson-Kane, E.G., Hunt, M., Harper, D.N., 2002. Rats demand social contact. Anim. Welf. 11, 327-332.

Richardson, R., Campbell, B.A., 1988. Effects of home nest odors on black-white preference in the developing rat: implications for developmental learning research. Behav. Neural Biol. 50, 361-366.

Richardson, C.A., Flecknell, P.A., 2005. Anaesthesia and post-operative analgesia following experimental surgery in laboratory rodents: are we making progress? Altern. Lab. Anim. 33, 119-127.

Saibaba, P., Sales, G.D., Stodulski, G., Hau, J., 1996. Behaviour of rats in their home cages: daytime variations and effects of routine husbandry procedures analysed by time sampling techniques. Lab. Anim. 30, 13-21.

Schnecko, A., Witte, K., Lemmer, B., 1998. Effects of routine procedures on cardiovascular parameters of SpragueDawley rats in periods of activity and rest. J. Exp. Anim. Sci. 38, 181-190.

Schoeb, T.R., Davidson, M.K., Lindsey, J.R., 1982. Intracage ammonia promotes growth of Mycoplasma pulmonis in the respiratory tract of rats. Infect. Immun. 38, 212-217.

Schondelmeyer, C.W., Dillehay, D.L., Webb, S.K., Huerkamp, M.J., Mook, D.M., Pullium, J.K., 2006. Investigation of appropriate sanitization frequency for rodent caging accessories: evidence supporting less-frequent cleaning. J. Am. Assoc. Lab. Anim. Sci. 45, 40-43.

Serrano, L.J., 1971. Carbon dioxide and ammonia in mouse cages: effects of cage covers, population, and activity. Lab. Anim. Sci. 21, 75-85.

Sharp, J.L., Zammit, T.G., Azar, T.A., Lawson, D.M., 2002. Stress-like responses to common procedures in male rats housed alone or with other rats. Contemp. Top. Lab. Anim. Sci. 41, 8-14.

Studier, E.H., Baca, T.P., 1968. Atmospheric conditions in artificial rodent burrows. Southwest. Nat. 13, 401-410.

Tepper, J.S., Weiss, B., Wood, R.W., 1985. Alterations in behavior produced by inhaled ozone or ammonia. Fundam. Appl. Toxicol. 5, 1110-1118.

Voipio, H.M., Nevalainen, T., Halonen, P., Hakumäki, M., Björk, E., 2006. Role of cage material, working style and hearing sensitivity in perception of animal care noise. Lab. Anim. 40, 400-409. 